\title{
Experimental analysis of the precessing vortex core in a free swirling jet
}

\author{
Fulvio Martinelli $\cdot$ Andrea Olivani $\cdot$ Aldo Coghe
}

Published online: 20 April 2007

(C) Springer-Verlag 2007

\section{Erratum to: Exp Fluids DOI 10.1007/s00348-006-0230-x}

Due to a processing error, the presentation of Fig. 7a, b and the corresponding figure capture was incorrect.

The correct figure with legend is given here.

The online version of the original article can be found at http://dx.doi.org/10.1007/s00348-006-0230-x.

F. Martinelli $(\bowtie)$

Dipartimento di Ingegneria Aerospaziale,

Politecnico di Milano, Via La Masa, 34,

Milano 20158, Italy

e-mail: fulvio.martinelli@polimi.it

\section{A. Olivani - A. Coghe}

Dipartimento di Energetica, Politecnico di Milano, Via La Masa, 34, Milano 20158, Italy
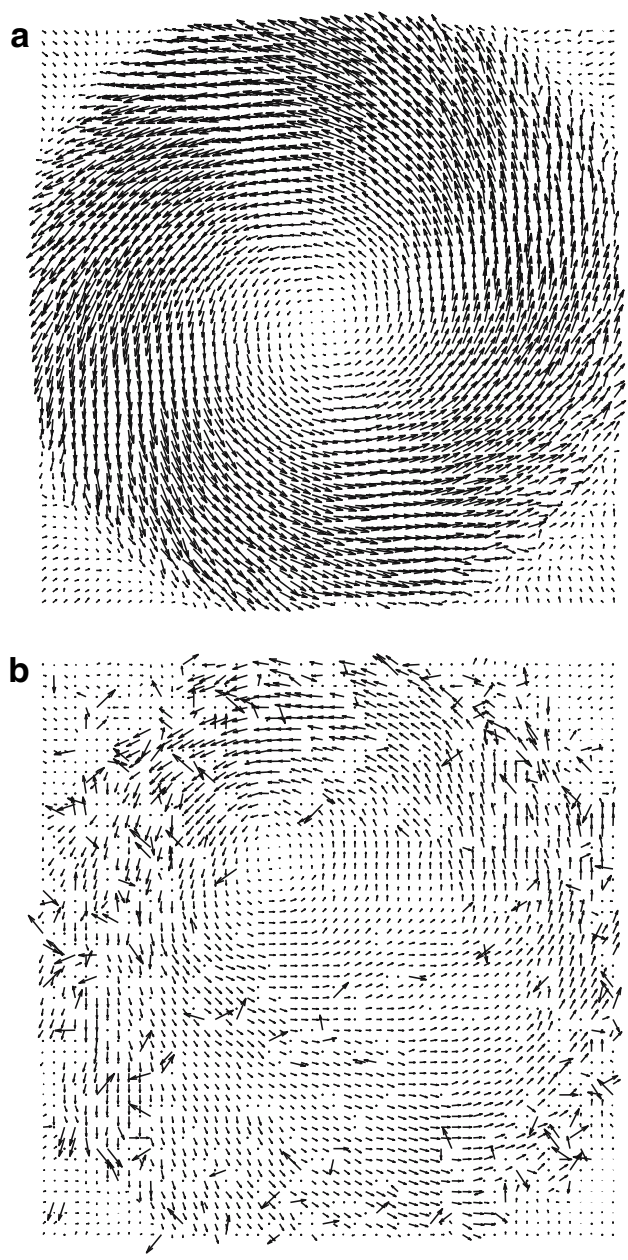

Fig. 7 Comparison between average and instantaneous velocity fields at the exhaust. a The mean flowfield, obtained from the averaging of 200 couples of images; $\mathbf{b}$ an instantaneous raw image, peak validated with no interpolation-Re $\approx 24,400, G \approx 2.76$. Erroneous vectors have not been removed from this figure to show the good quality of the instantaneous maps 